\title{
An investigation of the behaviour of planar jets using a time accurate fractional step scheme
}

\author{
$\begin{array}{ll}\text { P. L. Morgan* } & \text { S. W. Armfield }\end{array}$
}

(Received 1 October 2001; revised 14 January 2003)

\begin{abstract}
The transition behaviour of planar jets has been the subject of extensive investigation, using both numerical and experimental approaches. It is well known that above a critical Reynolds number the jet will exhibit a sinusoidal, flapping, bifurcation. Numerical simulations of planar jet flow have been carried out using a time accurate fractional step NavierStokes solver defined on a non-staggered grid. Results have been obtained for a range of Reynolds numbers to investigate the bifurcation and stability characteristics of the flow and to obtain the critical Reynolds number for the onset of unsteadiness. This critical Reynolds number is found to be
\end{abstract}

*School of Aerospace, Mechanical and Mechatronic Engineering, University of Sydney, Sydney, Australia. mailto:pmorgan@aeromech.usyd.edu. au

†as above. mailto:armfield@mech.eng. usyd.edu.au

${ }^{0}$ See http://anziamj.austms.org.au/V44/CTAC2001/Morg for this article, (C) Austral. Mathematical Soc. 2003. Published 1 April 2003. ISSN 1446-8735 
in good agreement with that obtained experimentally and for a non-parallel Bickley jet using a semi-analytic stability technique, whereas earlier parallel stability results considerably under-predict this value. The numerical method is described and results presented detailing the character and behaviour of the steady and bifurcated flow together with comparisons to previous investigations.

\section{Contents}

1 Introduction

C551

2 Governing equations

C553

3 Numerical method

C554

4 Results

C558

5 Discussion

C564

6 Concluding remarks

C566

References

C566

\section{Introduction}

The planar jet is an evolving coherent structure where the two shear layers containing vortices of opposing sign encapsulate an irrotational core that has a length of approximately three times that of the inlet height. The steady plane horizontal jet velocity along the centreline in the $x$ direction diminishes like $x^{-1 / 3}$ while the jet 
spreads in the $y$ direction like $x^{2 / 3}$, and has a symmetric $\operatorname{sech}^{2} y$ profile as shown analytically by Schlichting [15] in 1933. The interaction downstream of these vortices leads to two possible unstable bifurcation modes. However, all jets exhibit evolving coherent structures, and their development stems from an initial perturbation, that of the fundamental frequency $f_{o}[9,5,10,16]$. The energy content of this wave increases until completely saturated, at which point an energy transfer takes place and the first harmonic begins to grow and ultimately becomes the new fundamental or dominant frequency [10]. These successive perturbations continue as the jet progresses downstream. In non-stratified environments the planar jet's initial fundamental frequency is parametrised only by the Reynolds number. At a super critical Reynolds number the jet will exhibit an unsteady bifurcation with a characteristic mode of instability.

There has been extensive research compiled on flow characteristics and preferred modes of stability of two dimensional jets. Sato [14] found experimentally that two kinds of instability occur when the laminar flow field becomes unstable, and that these instabilities were predominantly dependent on the jet's inlet velocity profile. A uniform jet inlet velocity profile was found to be associated with symmetric or varicose mode instability, whilst a fully developed parabolic jet inlet profile was found to be associated with asymmetric or sinuous mode instability. However observations of the near field planar homogeneous jet at slightly greater than the critical Reynolds number, by Beavers and Wilson [4], showed that the natural breakdown of the jet was essentially symmetric for low Reynolds number and that the instability and vortex formation were sensitive to external perturbations irrespective of the inlet velocity profile.

In this paper the time dependent behaviour of a planar jet is investigated for a range of Reynolds numbers. For low, sub-critical, Reynolds numbers the jet is steady and symmetric, while for higher 
Reynolds numbers the jet becomes asymmetric and unsteady. A second order in time fractional step scheme defined on a non-staggered grid is used for the numerical simulations. The paper is organised as follows: In Section 2 the governing equations and domain are given with the appropriate boundary conditions for the planar horizontal jet. The non-staggered fractional step method is discussed in detail in Section 3. Simulation results are given in Section 4 and concluding remarks presented in Section 5.

\section{Governing equations}

The governing equations for an incompressible flow are the NavierStokes equations,

$$
\begin{aligned}
\mathbf{u}_{t}+(\mathbf{u} \cdot \nabla) \mathbf{u} & =-\nabla P+\frac{1}{\operatorname{Re}} \nabla^{2} \mathbf{u}, \\
\nabla \cdot u & =0,
\end{aligned}
$$

where $P$ is the pressure, $\mathbf{u}$ is the velocity vector field, $\operatorname{Re}=\bar{U} L / \nu$ is the Reynolds number with $\bar{U}$ being a characteristic velocity scale, $L$ a characteristic length scale and $\nu$ the kinematic viscosity of the fluid.

The discretised equations are solved in the domain $0 \leq y \leq \mathrm{ER}$, $0 \leq x \leq 20$, and $t \geq 0$, where ER is the expansion ratio defined as $Y / H$, with $Y$ the dimensional height of the domain and $H$ the dimensional jet inlet height. The domain size and jet inlet height was chosen to approximately match an experimental configuration used by Sato [14]. The top, bottom and left boundaries, with the exception of the jet inlet, are impervious with zero velocity, while the right boundary is open with zero normal velocity gradient. The boundary conditions along with the general model configuration are shown in Figure 1. Initially the fluid is quiescent with $u=v=0$. 


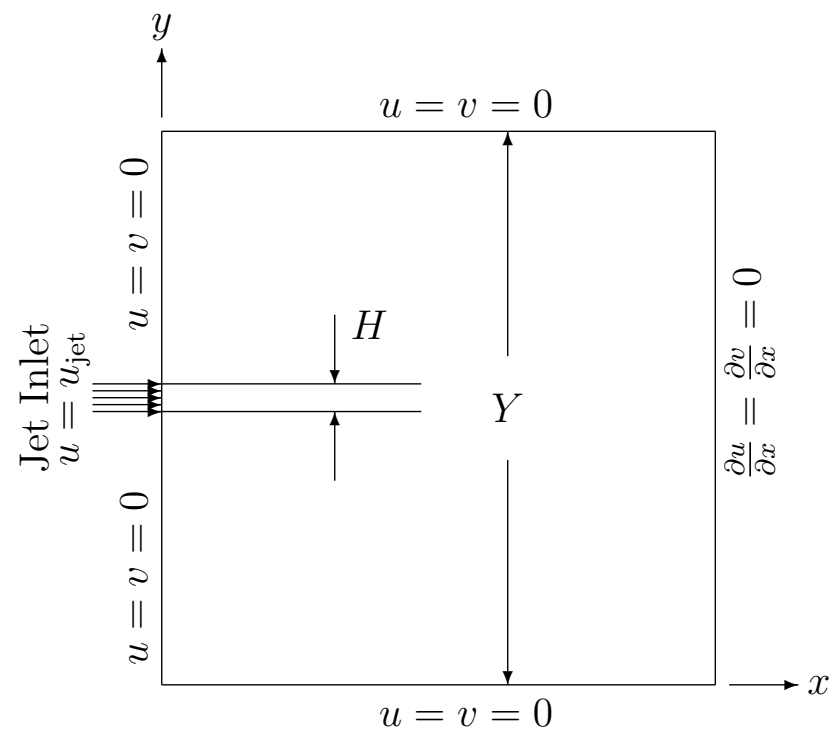

Figure 1: Geometry configuration and boundary conditions

As shown the computational domain is a rectangular box. All the lengths are non-dimensionalised by the jet inlet height $H$, with the jet located at the centre $(\mathrm{ER} / 2)$ of the left hand side of the box. The non-dimensional inlet velocity $u_{j e t}$ has a parabolic profile with maximum set to unity.

\section{$3 \quad$ Numerical method}

The governing equations are solved using a second-order time accurate fractional step Navier-Stokes solver. The domain is discretised on a non-staggered grid using finite volumes with no nodes lying on the boundaries. The boundary values are set by including an extra point outside the boundary with a prescribed value. The value on the boundary is then the average of this outside node 
and its closest neighbor. When values of dependent variables are required at locations other than the nodes, linear interpolation is used. The viscous, pressure gradient and divergence terms are approximated using three point second-order central differencing and the advection terms are discretised using the QUICK third-order upwind scheme [12]. Time integration is achieved using an explicit Adams-Bashforth scheme for the advection terms and an implicit Crank-Nicolson scheme for the diffusive terms resulting in the following second-order in time system:

$$
\begin{aligned}
& \frac{v^{n+1}-v^{n}}{\Delta t}+\left[\frac{3}{2} \mathcal{H}\left(v^{n}\right)-\frac{1}{2} \mathcal{H}\left(v^{n-1}\right)\right] \\
& =-\mathcal{G} p^{n+\frac{1}{2}}+\frac{1}{2 \operatorname{Re}} \mathcal{L}\left(v^{n+1}+v^{n}\right) ; \\
& \mathcal{D} v^{n+1}=0 .
\end{aligned}
$$

Here the discrete velocity and pressure are represented by $v$ and $p$ and the superscript $n$ represents time location. The discrete advection operator, the discrete gradient, the discrete Laplace operator and the discrete divergence are represented by $\mathcal{H}, \mathcal{G}, \mathcal{L}$ and $\mathcal{D}$ respectively [1].

Fractional step methods have become popular in recent years, especially in the simulation of unsteady flows, and are therefore suitable methods for investigating the transitional nature of the planar jet. In fractional step methods the pressure is used to enforce continuity, which is the characteristic role of pressure in incompressible flows. In this method to obtain an initial approximation of the velocity field, an integration is performed on an incomplete form of the momentum equations. The resulting velocity field may not necessarily be divergence free so a correction in the form of an orthogonal projection of the velocity field onto a divergence free solution is made, leaving the vorticity unchanged. The fractional step projection method of Kim and Moin [13] drops the pressure gradients 
from the momentum equations and enforces specialised boundary conditions on the intermediate velocity field to obtain second order in time accuracy. The intermediate velocity is then corrected using a pressure obtained by solving a Poisson equation with the divergence of the intermediate velocity field on the right hand side.

Armfield and Street [2] use an alternative method which is similar in nature to Kim and Moin's method, but with the previous time step pressure gradient included in the momentum equations. A Poisson equation is then solved to obtain a pressure correction which is used to correct the intermediate velocity field and enforce continuity.

In this procedure the correct $n+\frac{1}{2}$ level pressure is not available to use in the integration of equation (3), and thus an approximation $v^{\star}$ is obtained for $v^{n+1}$ by integrating equation (3) with $p^{n-1 / 2}$, the previous time level pressure. This approximation will not in general satisfy continuity, and thus a correction is performed,

$$
v^{n+1}=v^{\star}-\Delta t \mathcal{G P},
$$

where $\mathcal{P}$ is the pressure correction and $v^{n+1}$ satisfies continuity. An equation for $\mathcal{P}$ is obtained by substituting equation (5) into equation (4) to give,

$$
\mathcal{L} \mathcal{P}=\frac{\mathcal{D} v^{\star}}{\Delta t} .
$$

Once $\mathcal{P}$ is obtained, the velocity is then corrected and the pressure updated using the pressure correction:

$$
p^{n+\frac{1}{2}}=p^{n-\frac{1}{2}}+\mathcal{P} .
$$

This integration and correction procedure is then repeated at each consecutive time step. Armfield and Street have compared this method and analysed several other fractional step methods on staggered and non-staggered grids, their findings demonstrate the ad- 
vantages of the pressure correction approach in terms of increased efficiency whilst retaining overall second order accuracy $[1,2]$.

In the $x$ direction a non-uniform grid is used with the smallest cell size set to $\Delta x=0.038$ located at $x=0$, expanded by a stretching factor of 1.10 . In the $y$ direction the jet inlet region is spanned by a uniform grid with $\Delta y=0.03$. The grid is then stretched towards the upper and lower boundaries, contracting again at the boundaries with $\Delta y=0.03$ adjacent to each boundary, with maximum stretching and minimum contraction factors of 1.05 and 0.95 respectively. This configuration results in $195 \times 186$ nodes in the $x$ and $y$ directions, with 33 nodes spanning the jet inlet.

Grid dependency tests were carried out on fine and coarse mesh configurations and their solutions compared with the solution obtained for the mesh outlined above. The fine mesh has a grid size of $257 \times 276$ nodes in the $x$ and $y$ directions respectively, and 36 nodes spanning the inlet. The smallest cell size in the $x$ direction being $\Delta x=0.027$, and the smallest cell size in the $y$ direction being $\Delta y=0.027$. The coarse mesh has a grid size of $44 \times 115$ nodes in the $x$ and $y$ directions respectively, and 13 nodes spanning the inlet. The smallest cell size in the $x$ direction being $\Delta x=0.3$, and the smallest cell size in the $y$ direction being $\Delta y=0.1$. Three time steps were tested and compared for all mesh configurations, $\Delta \tau=0.05, \Delta \tau=0.01$ and $\Delta \tau=0.001$ with $\Delta \tau=0.01$ used for this investigation. Grid and time step dependency results show negligible variation in the flow pattern characteristics and critical Reynolds numbers. 


\section{Results}

A typical planar jet flow has three distinct regions; the near field, the transitional region and the far field fully developed or self-preserving region. In the near field at $x=0$, the flow is dominated by the interaction of the jet and the fluid entrained from the ambient by two large vortices. These vortices combine to form a coherent structure that progresses downstream with constant momentum flux and increasing mass flux. The jet instability is investigated by obtaining solutions for a range of Reynolds numbers and examining the time series obtained at the centre of the downstream boundary. The critical Reynolds number is defined to be the lowest Reynolds number at which unsteady behaviour is observed in the time series.

In Figure 2 the velocity vector field is shown for a sub-critical jet with Reynolds number, $\mathrm{Re}=21$ at $t=200$ and $\mathrm{ER}=21$. The vector plot shows results at every second grid node location. The jet can be seen entering the domain from the left hand side and exiting at the right side. The figure clearly shows a symmetric flow pattern in the transverse direction with the jet entraining fluid from the ambient and the establishment of two strong vortex pairs of opposing sign which are located approximately at $x=10$. This flow was found to be steady.

The vector plots of a super-critical jet are shown in Figure 3 with Reynolds number, $\operatorname{Re}=28$ at $t=150$ and at $t=160$. In this figure the jet has a periodic sinusoidal like bifurcating motion, the instability mode which is expected for a parabolic jet inlet velocity profile [14]. At $t=150$ the figure shows a strong vortex located at $x=9, y=16$ with the other weaker vortex located at approximately $x=19, y=9$. At $t=160$ the previous strong vortex has weakened and is now located at $x=18, y=11$, whilst the other vortex has become the stronger and is located at $x=8, y=5$. Af- 


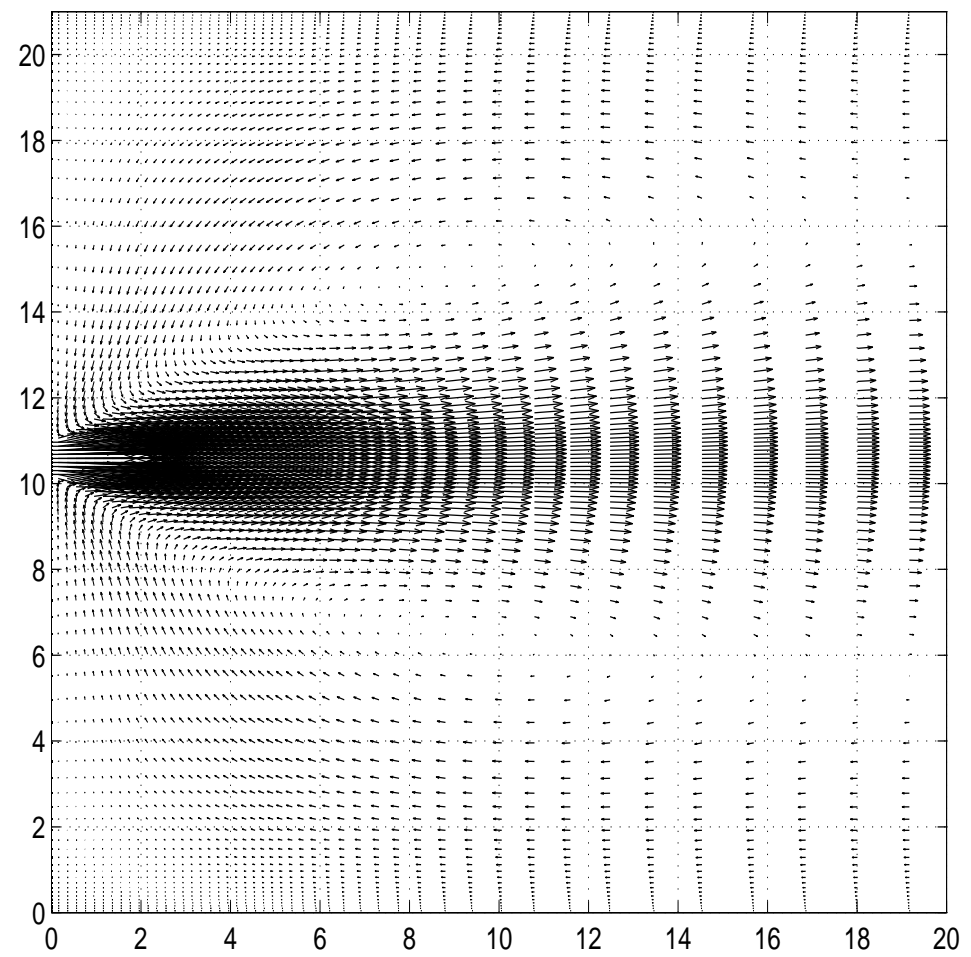

Figure 2: Velocity vectors for $\mathrm{Re}=21, \mathrm{ER}=21$ at $t=200$. 

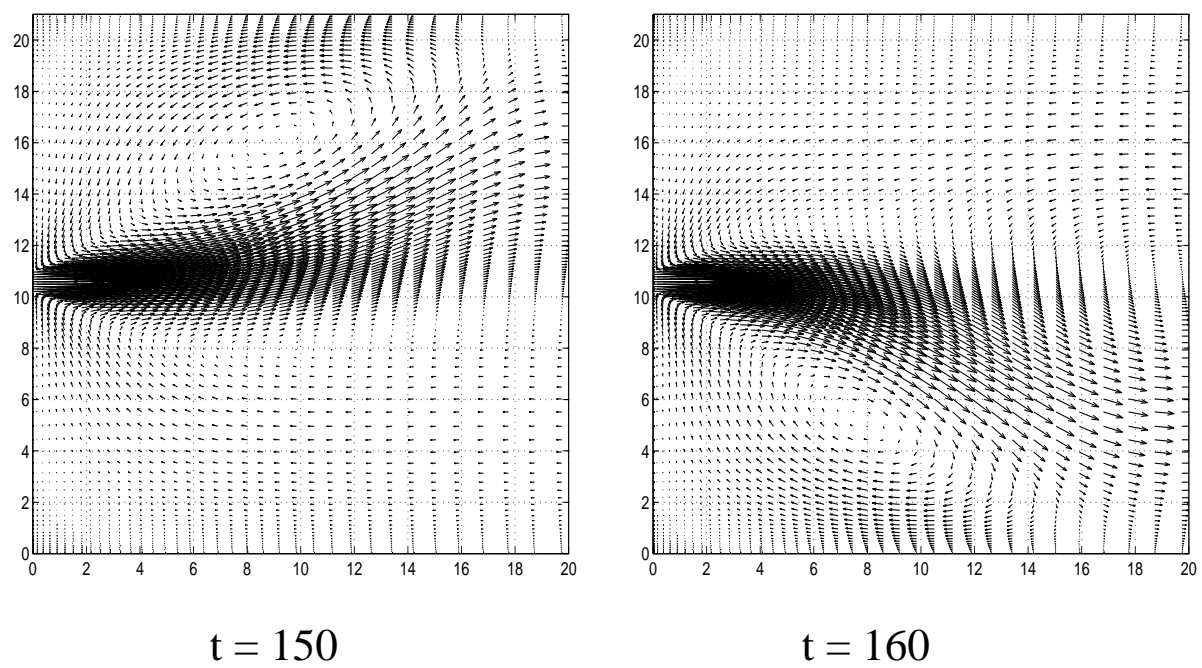

FIgURE 3: Velocity vectors for $\mathrm{Re}=28, \mathrm{ER}=21$

ter $t=160$, the flow pattern repeats with the stronger/weaker vortex pairs appearing at approximately the same locations they were at $t=150$. Fully developed flow is ultimately established with a periodic sinusoidal motion. The axial velocity along the jet centreline for $\mathrm{Re}=28$ and $\mathrm{ER}=21$ is shown in Figure 4. This figure contains the peak horizontal velocity value at 20 equally spaced $x$ locations and shows that the jet axial velocity clearly has an $x^{-1 / 3}$ form.

In Figure 5 the $x$ velocity component time series are given for $\mathrm{Re}=22.5,23,24,26$ at $x=20, y=10.5$. All results presented display a periodic bifurcation with an increase in amplitude observed with increasing Reynolds number, although the Re $=22.5$ signal is not discernible at this scale. Discrete Fourier Transforms were obtained for time series $\mathrm{Re}=21,21.5,22,22.5,23,23.5$ and are shown in Figure 6, to allow accurate identification of the minimum Re at which unsteady flow occurs. 


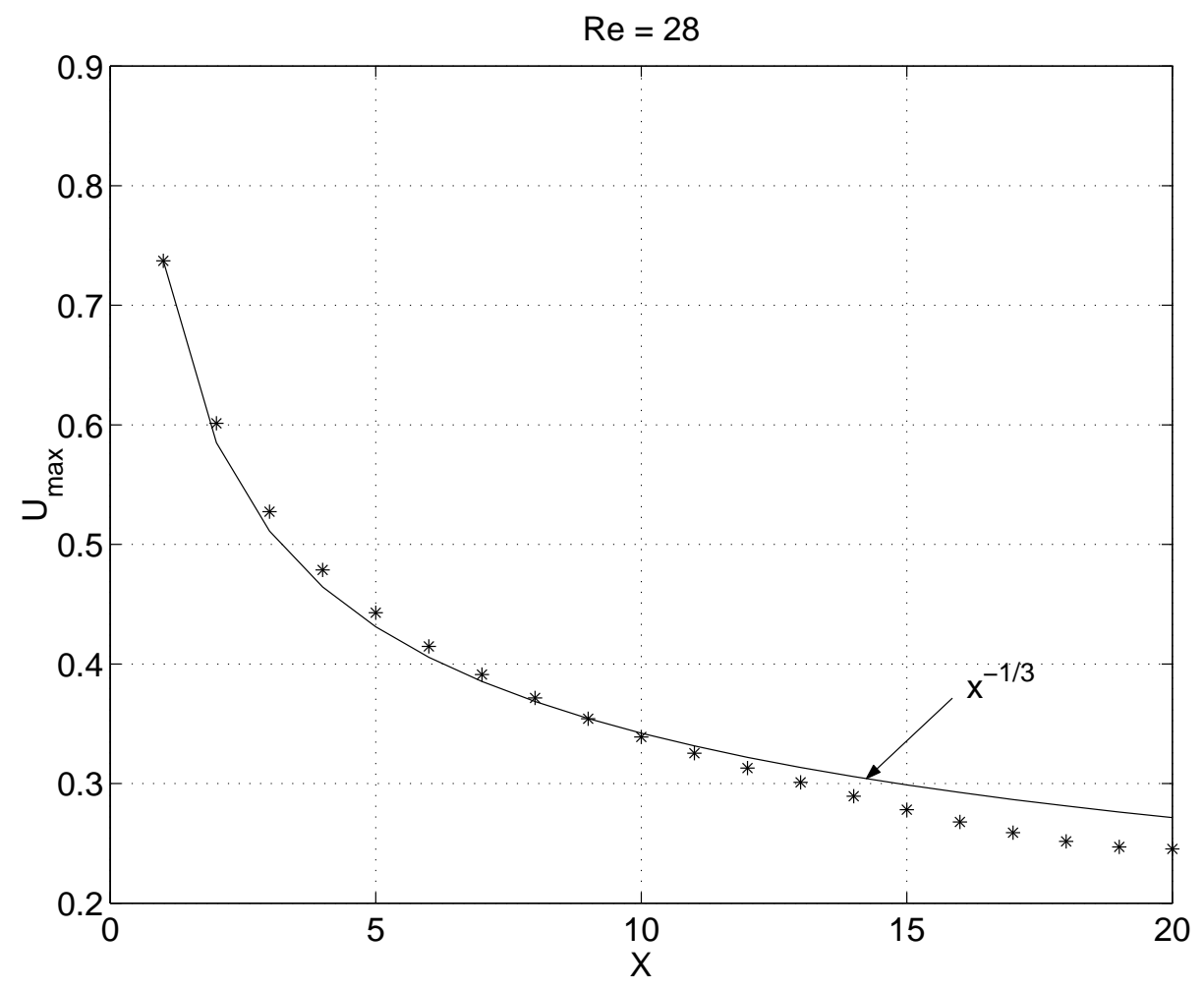

FiguRE 4: Jet growth rate in the $x$ direction, $\mathrm{ER}=21$ 


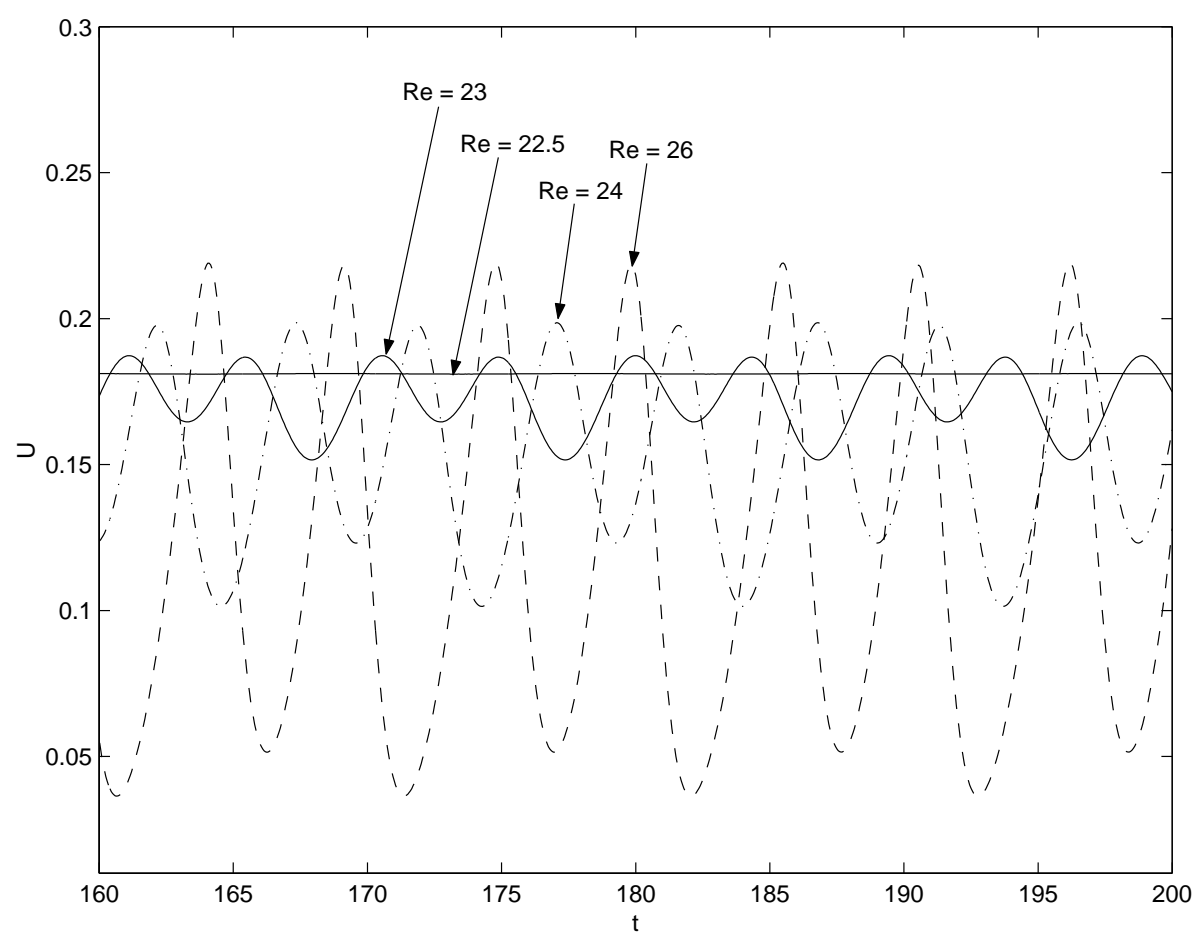

FiguRE 5: $x$ velocity time series for $\mathrm{Re}=22.5,23,24,26, \mathrm{ER}=21$ at $x=20, y=10.5$ 

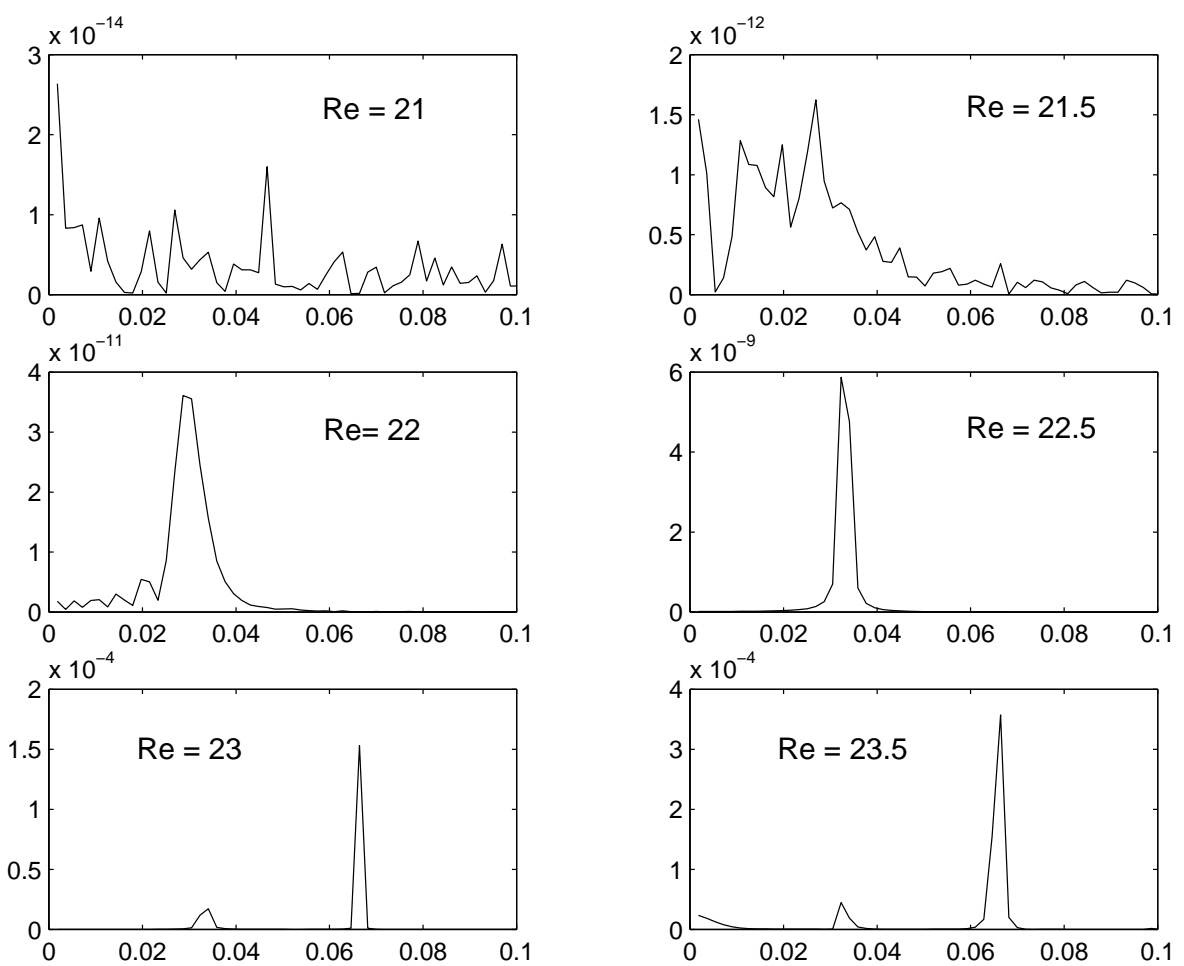

FiguRE 6: FFT results for $\mathrm{Re}=21,21.5,22,22.5,23,23.5, \mathrm{ER}=21$ with temporal wave-number on the horizontal axis.

The $\mathrm{Re}=21$ result shows a signal with no discernible peak and an amplitude of order $10^{-14}$. This is of the order of numerical round off for double precision arithmetic and the result is therefore classified sub-critical. The $\mathrm{Re}=21.5$ result is similarly classified as sub-critical. At $\mathrm{Re}=22$ a distinct peak is observed representing a fundamental frequency with amplitude of order $10^{-11}$ occurring at a temporal wave-number $\alpha=0.03$. Visual inspection of the time series, flow contours and velocity vectors indicate that at this critical 
Reynolds number the jet has a periodic bifurcating motion, and this is therefore classified as the critical Reynolds number. At $\operatorname{Re}=$ 22.5 a signal with amplitude of order $10^{-9}$ is seen at $\alpha=0.0325$. At $\operatorname{Re}=23$ the signal has an amplitude of order $10^{-4}$ with $\alpha=$ 0.035 ; however, an additional larger amplitude signal is seen at $\alpha=$ 0.065 . This new signal, with exactly double the wave-number of the base signal, is the first harmonic and occurs when the amplitude of the base signal reaches the maximum that may be attained for this configuration. Energy from the base mode is then transferred to the first harmonic. This effect is referred to as saturation of the base frequency. As the Reynolds number is further increased the amplitude of the base mode remains approximately constant, while that of the first harmonic continues to increase.

\section{Discussion}

The results presented above show that the critical Reynolds number for the occurrence of unsteadiness for a homogeneous jet with parabolic inlet flow in an enclosure with $\mathrm{ER}=21$ and length $=20$ is $\operatorname{Re}_{\text {crit }} \cong 22$. Additional results have been obtained for the same jet in enclosures of the same length with expansion ratios $\mathrm{ER}=3$, 5 and 80. These results are presented in Table 1, together with the results of: Garg [8], obtained using a non-parallel stability analysis; Drazin and Reid [6], obtained using a parallel stability analysis; the experimental results of Sato [14]; and a series of results obtained using direct numerical simulation. The results obtained here show that the critical Reynolds number decreases with increasing ER, with the $\mathrm{ER}=21$ result being comparable to the $\mathrm{ER}=80$ result. On this basis we suggest that the ER $=80$ result is a good approximation of the infinite expansion ratio result. Also observe that the $\mathrm{ER}=21$ result is in good agreement with the non-parallel sta- 
TABLE 1: Critical Reynolds numbers, $\mathrm{Re}_{\text {crit }}$.

\begin{tabular}{|l|l|}
\hline Present investigation: & 18 \\
$\mathrm{ER}=80$ & 22 \\
$\mathrm{ER}=21$ & 26 \\
$\mathrm{ER}=5$ & 50 \\
$\mathrm{ER}=3$ & \\
Stability theory: & 21.6 \\
Non-parallel Theory (Garg [8]) & 4 \\
Parallel Theory (Drazin \& Reid [6]) & \\
Experimental: & $\sim 20$ \\
ER $=20$, Experimental (Sato [14]) & \\
Other numerical simulations: & $\sim 50$ \\
ER $=20$, FEA (Sarma et al. [11]) & $27-30$ \\
ER $=5$, ENTWIFE FEA (Battaglia et al. [3]) \\
ER $=3$, ENTWIFE FEA (Battaglia et al. [3]) & $57-58$ \\
ER $=3$, ENTWIFE FEA (Fearn et al. [7]) & $\sim 47$ \\
ER $=3$, ENTWIFE FEA, previous version (Fearn & 54 \\
et al. [7]) & \\
\hline
\end{tabular}

bility result of Garg and the experimental result of Sato. The FEA result of Sarma for $\mathrm{ER}=20$ is approximately twice this value. On the basis of the agreement between Garg, Sato and the present result we believe that $\operatorname{Re}_{\text {crit }} \sim 20$ is the correct value for the $\mathrm{ER}=21$ case. The poor agreement of the Drazin and Reid result may be attributed to the parallel assumption used in their stability analysis. Other results obtained by Battaglia et al. and Fearn et al. for smaller ER are in generally good agreement with the results obtained here. 


\section{Concluding remarks}

Results obtained using the fractional step method presented here have demonstrated the basic features and behaviour of homogeneous planar jet flow. Using time series collected at the centre of the downstream boundary was found to be a suitable means of determining transition to unsteady flow, providing a critical Reynolds number in good agreement with that obtained experimentally by Sato, and providing support for the non-parallel analysis of Garg. The time series results provided a further insight into the transient behaviour of the flow whereby upon saturation harmonics of the base frequency are amplified as the Reynolds number is increased for super critical flow.

Acknowledgements: This project was supported by the Australian Research Council through grant No. A00104152.

\section{References}

[1] S. W. Armfield and R. Street. An analysis and comparison of the time accuracy of fractional-step methods for the Navier-Stokes equations on staggered grids. Int. J. Comp. Physics(1999) 153, 660-665. C555, C557

[2] S. W. Armfield and R. Street. The fractional-step method for the Navier-Stokes equations on staggered grids: the accuracy of three variations. Int. J. Numer. Meth. Fluids(2002) 38, 255-282 C556, C557 
[3] F. Battaglia, S. J. Tavener, A. K. Kulkarni and C. L. Merkle. Bifurcation of low Reynolds number flows in symmetric channels. AIAA Journal(1997) 35, 99-105. C565

[4] G. S. Beavers and T. A. Wilson. Vortex growth in jets. J. Fluid Mech.(1970) 44, 97-112. C552

[5] J. Cohen and I. Wygnanski. The evolution of instabilities in the axisymmetric jet. Part 1 . The linear growth of disturbances near the nozzle. J. Fluid Mech.(1987) 176, 191-219. C552

[6] P. G. Drazin and W. H. Reid. Hydrodynamic stability. Cambridge University Press(1981) C564, C565

[7] R. M. Fearn, T. Mullin and K. A. Cliffe. Non-linear flow phenomena in a symmetric sudden expansion. J. Fluid Mech.(1990) 211, 595-608. C565

[8] V. K. Garg. Spatial stability of the non-parallel Bickley jet. J. Fluid Mech(1981) 102, 127-140. C564, C565

[9] C. Ho and P. Huerre. Perturbed Free Shear Layers. Ann. Rev. Fluid Mech.(1984) 16, 365-424. C552

[10] F. Hsiao and J. Huang. On the evolution of instabilities in the near field of a plane jet. Phys. Fluids A(1989) 2, 400-412. C552

[11] A. S. R. Sarma, T. Sundararajan and V. Ramjee. Numerical simulation of confined laminar jet flows. Int. J. Numer. Meth.(2000) 33, 609-626. C565

[12] B. P. Leonard, A stable and accurate convective modelling procedure based on quadratic upstream interpolation. Comput. Meth. Appl. Mech. Eng.(1979) 19, 59-98. C555 
[13] J. Kim and P. Moin Applications of a fractional step method to incompressible Navier-Stokes equations. J. Comput. Phys.(1985) 59, 308-323. C555

[14] H. Sato. The stability and transition of a two-dimensional jet. J. Fluid Mech.(1960) 7, 53-80. C552, C553, C558, C564, $\mathrm{C} 565$

[15] H. Schlichting. Boundary-layer Theory, 7th Ed. McGraw-Hill, Inc.(1979) C552

[16] F. O. Thomas and K. M. K. Prakash. An experimental investigation of the natural transition of an untuned planar jet. Phys. Fluids A(1990) 3, 90-105. C552 